\title{
Relação entre força muscular respiratória e funcionalidade em idosos residentes em instituições de longa permanência
}

\section{Relation ship between respiratory muscle strength and function in elderly residents in institutions of long stay}

\author{
Cleide Cristina Firmino Oliveira, Jamine Barros Oliveira Araújo, \\ Luciana Araújo dos Reis
}

\section{Como citar este artigo:} OLIVEIRA, CLEIDE C. F.; ARAÚJO, JAMINE B. O.; REIS, LUCIANA A. Relação entre força muscular respiratória e funcionalidade em idosos residentes em instituicões de longa permanência. Revista Saúde (Sta. Maria). 2020; 46 (2).

\section{Autor correspondente:}

Nome: Luciana Araújo dos Reis E-mail: lucianauesb@yahoo.com.br Formação Profissional: Doutora pela Universidade Federal do Rio Grande do Norte, Natal, RN, Brasil. Filiação Institucional: Universidade estadual do Sudoeste da Bahia/ Faculdade Independente do Nordeste.

Filiação Institucional: Faculdade Independente do Nordeste

Endereço para correspondência: Av. Luis Eduardo Magalhães

Bairro: Candeias

Cidade: Vitória da Conquista

Estado: Bahia

Data de Submissão:

10/02/2020

Data de aceite:

21/09/2020

Conflito de Interesse: Não há conflito de interesse

\section{(cc) BY-NC-ND}

\section{RESUMO}

No envelhecimento ocorre uma redução gradual da força muscular respiratória e da capacidade funcional, nesta perspectiva, o presente estudo tem por objetivo identificar a relação entre a força muscular respiratória e a funcionalidade em idosos residentes em instituições de longa de permanência. Trata-se de um estudo do tipo analítico com delineamento transversal e abordagem quantitativa, tendo como campo de pesquisa três instituições de longa permanência para idosos nos municípios de Vitória da Conquista e Jequié/BA, com amostra de 62 idosos. 0 instrumento de pesquisa foi constituído de cinco (5) partes: avaliação cognitiva, informações sociodemográficas, estado de saúde, força muscular respiratória (PImáx e PEmáx) e capacidade funcional (atividades básicas e instrumentais de vida diária). Os dados foram analisados por meio da análise estatística descritiva e aplicação do coeficiente de correlação de Spearman. Verificou-se que houve diferença estatística significativa entre PEmáx e atividades instrumentais de vida diária $(p=0,000)$. Os resultados encontrados no presente estudo sugerem que os valores reduzidos da PEmáx estão associado ao comprometimento das atividades instrumentais de vida diária.

PALAVRAS-CHAVE: Idosos; Funcionalidade; Força muscular respiratória.

\section{ABSTRACT}

Aging in a gradual reduction in respiratory muscle strength and functional capacity occurs, in this perspective, this study aims to identify the relationship between respiratory muscle strength and function in elderly residents of long-stay institutions. This is a study of the analytical type with cross design and quantitative approach, taking as a research field three institutions for the aged in the cities of Vitória da Conquista and Jequie/BA with a sample of 62 elderly. The survey instrument consisted of five (5) parts: cognitive assessment, sociodemographic information, health status, respiratory muscle strength (MIP and MEP) and functional capacity (basic and instrumental activities of daily living). Data were analyzed using descriptive and application of the Spearman correlation coefficient statistical analysis. It was found that there was statistically significant difference between MEP and instrumental activities of daily living $(p=0.000)$. The results of this study suggest that the reduced values of MEP are associated with impairment of instrumental activities of daily living.

KEYWORDS: Elderly; functionality; Respiratory muscle strength. 


\section{INTRODUÇÃO}

Hoje o envelhecimento populacional tem sido realidade na maioria das sociedades, é fato: o mundo está envelhecendo. A Organização das Nações Unidas (ONU) prevê uma transição demográfica sem precedentes, e que daqui a 2050 o número de pessoas acima de 60 anos ou mais poderá chegar a 2 bilhões em todo o mundo, a maior parte delas vivendo nos países em desenvolvimento ${ }^{1}$. Situação que também se mostra no contexto brasileiro, no qual se estima que, em 2020, será o $6^{0}$ país no ranking mundial, num rápido crescimento e modificação da estrutura da pirâmide etária².

O envelhecimento populacional mundial traz inúmeras complicações nas condições de saúde. A transição epidemiológica associada à transição demográfica tem se revelado um novo panorama nos indicadores de saúde e no perfil de morbimortalidade. Nesse contexto há uma tendência de crescimento do número de idosos, porém apresentando maiores condições crônicas. Dessa forma, as doenças crônicas são consideradas prejudicais a manutenção da saúde e da qualidade de vida dos idosos, tendo estas uma grande relação com uma maior incapacidade funcional ${ }^{3}$. Com base nos valores apresentados pelo Instituto Brasileiro de Geografia e Estatística, a população com idades superiores a 60 anos em 1994 representava apenas 4\%, no ano 2000 esse percentual aumentou para 8,6 \%, chegando a 7,4\% em 2010, tendo tendência de elevação desses valores no ano 2020 para 13\% de idosos do total da população brasileira4.

O processo de envelhecimento acompanhado da longevidade, somado às doenças crônico-degenerativas e as alterações fisiológicas que acompanham o idoso, podem provocar um declínio considerável na capacidade respiratória e capacidade funcional dos idosos. Estudos voltados para a função respiratória no idoso apontam um declínio considerado pequeno nos primeiros anos da terceira idade e acelerado com o avançar da idade ${ }^{5}$.

Neste contexto o envelhecimento traz consigo dependência do cuidado de outros, e como alguns idosos não possuem essa possibilidade de cuidado no seio familiar, a única opção é a moradia em instituição de longa permanência ${ }^{5-6}$.

A avaliação da relação da força dos músculos respiratórios coma capacidade funcional é de grande relevância, uma vez que favorece a introdução de intervenções que venham melhorar as condições dos idosos na sua vida diária, melhorando sua funcionalidade e consequentemente a sua qualidade de vida. Nesta perspectiva, o presente estudo tem por objetivo identificar a relação entre força muscular respiratória e a capacidade funcional em idosos residentes em instituições de longa permanência.

\section{MATERIAL E MÉTODOS}

Trata-se de uma pesquisa do tipo analítica com delineamento transversal e abordagem quantitativa. A pesquisa foi desenvolvida em três instituições de longa permanência para idosos nos municípios de Vitória da Conquista e Jequié, 
situados no interior do Estado da Bahia. Trata-se de uma instituição de longa permanência de caráter filantrópico, que se mantém através de doações da comunidade e da própria aposentadoria dos idosos, que possuem por objetivo atender ao idoso sem vínculo familiar ou sem condições de prover a própria subsistência, e ainda ao idoso com vínculo familiar abandonado de modo a satisfazer as suas necessidades de moradia, alimentação, saúde e convivência social, regendo a vida do mesmo por meio de normas específicas.

A amostra do estudo foi constituída por todos os idosos residentes nas instituições de longa permanência com condições mentais para responder ao instrumento da pesquisa, sendo o estado mental avaliado pelo mini mental (MEEM), perfazendo um total de 62 idosos.

Os critérios de inclusão no estudo foram: os idosos que apresentaram capacidade cognitiva preservada para responder ao instrumento da pesquisa e não apresentassem condições físicas limitantes as quais impossibilitassem a avaliação da força muscular respiratória. Os critérios de exclusão no estudo foram idosos submetidos a cirurgias nas seis semanas anteriores à entrevista.

Os seguintes instrumentos foram utilizados:

- $\quad$ Avaliação cognitiva: utilizou-se o MEEM7. Instrumento útil para

detectar o déficit cognitivo nos idosos, composto por dez perguntas as quais avaliam memória a curto e longo prazo, orientação, informação do cotidiano e a capacidade de calcular. Através de perguntas do Mini-exame de estado mental. Na qual, a partir das respostas pode-se saber se o indivíduo está apto para o restante da pesquisa.

- Informações sóciodemográficas: foram avaliadas, sexo (masculino ou feminino); idade (coletada em anos completos); estado civil (sem companheiro, Casado/amasiado/namorando, solteiro, separado/desquitado/ divorciado, viúvo, não sabe/ não respondeu); tipo de renda; valor da renda; escolaridade (coletada em anos completos de estudo) e profissão.

- $\quad$ Estado de saúde: Foram avaliados: presença e tipos de problemas de saúde; presença e tipos de seqüelas; realização de tratamento; uso de medicamentos; auto percepção do estado de saúde; e avaliação da dor.

- $\quad$ Força muscular respiratória: Foi utilizado o Manovacuômetro (Famabras, Indústria Brasileira) escalonado em $-300 \mathrm{a}+300 \mathrm{~cm} \mathrm{H}_{2} \mathrm{O}$, para avaliar a força muscular respiratória pela medida das pressões Inspiratórias (PImáx) e expiratórias máximas (PEmáx). Para realização das manobras de PImáx e PEmáx, os idosos foram orientados 
a permanecer em posição sentada com um clipe nasal, que foi utilizado para evitar o escape de ar pela região nasal. A PImáx foi mensurada a partir do volume residual, ou seja, após uma expiração total. Os idosos foram orientados a realizar uma expiração máxima seguida de um esforço inspiratório máximo (a pesquisadora orientou a manobra dizendo "ponha todo o ar para fora, encha o peito de ar"). A PEmáx foi mensurada a partir da capacidade pulmonar total, ou seja, quando o volume de gás contido chegar ao máximo. Desta forma, os idosos foram orientados a realizar uma inspiração máxima e, em seguida, efetuar um esforço expiratório máximo (a pesquisadora orientou a manobra dizendo: "encha o peito de ar e sopre com força"). A determinação da PImáx e PEmáx foi realizada de acordo as diretrizes para os Testes de Função Pulmonar estipulado pela Sociedade Brasileira de Pneumologia e Tisiologia, seguindo os parâmetros de normalidade8, sendo realizada três medidas para cada.

- $\quad$ Capacidade funcional: Foram avaliadas as Atividades Básicas da Vida Diária/ABVD - Índice de Barthel e Atividades Instrumentais da Vida Diária/AIVD-Escala de Lawton. O Índice de Barthel é utilizado para avaliar capacidade funcional, sendo composto por 10 atividades: alimentação, banho, higiene pessoal, vestir-se, intestinos, bexiga, transferência para higiene íntima, transferência - cadeira e cama, deambulação e subir escadas. O escore correspondente à soma de todos os pontos obtidos, sendo considerado independente o indivíduo que atingir a pontuação total, isto é, 100 pontos. Pontuações abaixo de 50 indicam dependência em atividades de vida diária9. A Escala de Lawton engloba atividades mais complexas necessárias para uma vida social mais autônoma, tais como: telefonar, efetuar compras, preparar as refeições, arrumar a casa ou cuidar do jardim, fazer reparos em casa, lavar e passar a roupa, usar meios de transporte, usar medicação e controlar finanças particulares e/ou da casa. Para cada questão a primeira resposta significa independência, a segunda dependência parcial ou capacidade com ajuda ea terceira dependência. A pontuação máxima é 27 pontos $^{10}$.

As variáveis quantitativas foram apresentadas em média, desvio-padrão, valores mínimos e máximos. Já as variáveis categóricas foram calculadas as frequências e porcentagens. Para correlação entre capacidade respiratória (PImáx e PEmáx) e capacidade funcional (ABVD e AIVD) foi utilizado o coeficiente de correlação de Spearman, com nível de significância de 5\%. Os dados foram analisados no Programa Estatístico SPSS®, versão 21.0.

A pesquisa obedeceu às normas éticas, sendo aprovada pelo Comitê de Ética em Pesquisa com Seres Humanos, da Faculdade Independente do Nordeste (Protocolo $\left.n^{0} .26143514 .9 .0000 .5578\right)$. 


\section{RESULTADOS}

Contatou-se no presente estudo que houve uma maior distribuição de idosos do sexo feminino $(67,7 \%)$, solteiro (a) $(62,9 \%)$, aposentado (a) $(96,8 \%)$ e com faixa etária $\leq 74$ anos $(62,9 \%)$. A média de idade foi $74,56( \pm 7,5)$ anos e a média de anos estudados foi de $2,02( \pm 0,61)$ anos. As profissões mais freqüentes foram de doméstica $(25,8 \%)$, trabalhador rural $(16,1 \%)$ e bordadeira (12,9\%). Dados encontrados na tabela 1.

Tabela 1. Caracterização sociodemográfica dos idosos residentes em instituições de longa permanência. Vitória da Conquista /BA, 2019.

\begin{tabular}{clll}
\hline & N & $\%$ \\
\hline Sexo & & \\
& Masculino & 20 & 32,3 \\
& Feminino & 42 & 67,7 \\
\hline Estado Civil & & \\
& Casada (o) & 11 & 17,7 \\
& Solteiro (a) & 39 & 62,9 \\
& Viúvo (a) & 12 & 19,4 \\
\hline Faixa Etária & & \\
& $>74$ anos & 23 & 37,1 \\
& $\leq 74$ anos & 39 & 62,9 \\
\hline Renda & & \\
& Aposentado & 96,8 \\
& Pensão & 60 & 3,2 \\
\hline Total & 2 & 100,0 \\
\hline
\end{tabular}

Em relação às condições de saúde verificou-se que a maioria dos idosos apresentou uma boa percepção de saúde $(58,1 \%)$, presença de problemas de saúde $(90,3 \%)$, não possui sequelas $(51,6 \%)$, realiza tratamento $(72,6 \%)$ e apresenta dor (53,2\%). As patologias de maior frequência foram a Hipertensão arterial (19,4\%), Hipertensão arterial associada a Acidente vascular encefálico (19,4\%), Hipertensão arterial associada ao diabetes mellitus (16,1\%) e artrite (14,5\%). Dos idosos que apresentaram sequelas as de maior distribuição foram visão embasada $(21,0 \%)$ e deformidades de membros superiores e inferiores (19,4\%). Quanto ao tratamento os mais citados foram medicamentosos associado a fisioterapia $(41,9 \%)$ e medicamentoso (32,3\%). Dados encontrados na tabela 2. 
Tabela 2. Distribuição dos idosos residentes em instituições de longa permanência segundo as condições de saúde. Vitória da Conquista/BA, 2019.

\begin{tabular}{lll}
\hline & N & $\%$ \\
\hline Auto percepção de saúde & 2 & 3,2 \\
Excelente & 4 & 6,5 \\
Muito bom & 36 & 58,1 \\
Boa & 16 & 25,8 \\
Razoável & 4 & 6,5 \\
Péssima & & \\
Presença de problemas de saúde & 6 & 9,7 \\
Não & 56 & 90,3 \\
Sim & 32 & 51,6 \\
Presença de sequelas & 30 & 48,4 \\
Não & & \\
Sim & 17 & 27,4 \\
Realiza tratamento & 45 & 72,6 \\
Não & & \\
Sim & 33 & 53,2 \\
Presença de dor & 29 & 46,8 \\
Sim & 62 & 100,0 \\
Não & &
\end{tabular}

$\mathrm{Na}$ avaliação da capacidade funcional houve uma maior frequência de idosos classificados como dependentes tanto nas ABVD $(80,6 \%)$ quanto nas AIVD (96,8\%). Sendo que nas ABVD a maior parte dos idosos foi classificada com dependência leve $(77,8 \%)$ e nas AIVD a maioria dos idosos foi denominada com dependência parcial (95,2\%). Dados apresentados na Tabela 3.

Tabela 3. Distribuição dos idosos residentes em instituições de longa permanência segundo as condições de saúde. Vitória da Conquista /BA, 2019.

\begin{tabular}{ccc}
\hline & $\mathbf{n}$ & $\%$ \\
\hline Atividades Básicas de Vida Diária/ABVD & & \\
Dependente & 50 & 80,6 \\
Independente & 12 & 19,4 \\
\hline
\end{tabular}




\begin{tabular}{lll} 
Classificação das Atividades Básicas de Vida Diária & \\
Independente (100 pontos) & 12 & 19,4 \\
Dependência Leve ( $\geq 60$ pontos) & 49 & 77,8 \\
Dependência Moderada ( $\leq 40$ pontos) & 1 & 2.8 \\
\hline Atividades Instrumentais de Vida Diária/AIVD & 60 & 96,8 \\
$\quad$ Dependente & 2 & 3,2 \\
$\quad$ Independente & \\
\hline Classificação das Atividades Instrumentais de Vida Diária & 3,2 \\
$\quad$ Independente (27 pontos) & 2 & 96,8 \\
$\quad$ Dependência Parcial (26 a 10 pontos) & 60 & 100,0 \\
\hline Total & 62 & \\
\hline
\end{tabular}

Os valores médios da capacidade respiratória obtidos foram de PImáx foi de $18,63( \pm 16,75) \mathrm{cmH}_{2} \mathrm{O}$ e de PEmáx foi de $28,69( \pm 16,01) \mathrm{cmH}_{2} \mathrm{O}$. Dados encontrados na tabela 4 .

Tabela 4. Distribuição dos valores de força muscular (PImáx e PEmáx) dos idosos residentes em instituições de longa permanência. Vitória da Conquista /BA, 2019.

\begin{tabular}{llllll}
\hline & \multicolumn{5}{c}{ Valor Encontrado } \\
Capacidade Respiratória & $\mathbf{N}$ & Valor Previsto Média & Desvio-padrão IC (95\%) \\
\hline PImáx & 62 & $94,0( \pm 13,4)$ & 18,63 & 16,75 & 15,51 \\
PEmáx & 62 & $99,7( \pm 19,0)$ & 28,69 & 16,01 & 24,63 \\
\hline
\end{tabular}

Com a aplicação do coeficiente de correlação de Spearman verificou-se que houve diferença estatística significativa entre PEmáx e AIVD $(p=0,000)$. A análise mostrou uma correlação moderada, positiva e significativa entre 0 PEmáx e AIVD. As outras relações foram fracas e não significativas. Dados encontrados na tabela 5.

Tabela 5. Correlação entre a força muscular respiratória e a capacidade funcional. Vitória da Conquista /BA, 2019.

\begin{tabular}{lllll}
\hline & \multicolumn{4}{c}{ ABVD AIVD } \\
& $R$ & $P$ & $R$ & $P$ \\
\hline PImáx & $0,007^{1}$ & 0,959 & $0,195^{1}$ & 0,129 \\
PEmáx & $0,21^{1}$ & 0,101 & $0,468^{2}$ & 0,000 \\
\hline
\end{tabular}

\footnotetext{
${ }^{1}$ Correlação positiva fraca. $\quad{ }^{2}$ Correlação positiva moderada.
} 


\section{DISCUSSÃO}

No decorrer da pesquisa pôde-se observar uma maior frequência do sexo feminino e faixa etária menor ou igual a 74 anos. Tanto a distribuição entre as faixas etárias quanto a distribuição com relação ao sexo refletem a realidade do país, visto que a mulher figura em maior número na faixa etária acima de 60 anos ${ }^{11}$. A predominância de idosos pertencentes ao sexo feminino é devido a menor exposição a determinados fatores de risco, menor prevalência de tabagismo e uso de álcool, diferença quanto a postura em relação a doença e incapacidade e uma maior cobertura de assistência gineco-obstétrica ${ }^{12}$.

De acordo com o censo ${ }^{1}$, no município de Vitória da Conquista/BA havia 306.866 habitantes, sendo 147.879 homens e 158.987 mulheres, destes 30.593, (10\%) da população idosa têm idade acima de 60 anos. No município de Jequié, de acordo o censo ${ }^{1}$ apresenta 151.717 habitantes, sendo 73.612 homens e 78.105 mulheres, destes $17.330(11,1 \%)$ da população idosa com idade acima de 60 anos. Essa distribuição varia no Brasil de acordo com a região estudada.

Quanto ao índice de escolaridade a maioria dos idosos apresentou uma baixa média de anos estudados, o que é um indicativo ruim contra os riscos à saúde, uma vez que características, como analfabetismo, alterações do estado nutricional e aposentadoria, levam ao idoso uma maior dependência em relação a outras pessoas ${ }^{12}$.

Em relação à renda, verificou-se que a maior parte dos idosos apresentavam aposentadoria de 1 salário mínimo. Em estudo realizado no nordeste do Brasil, constatou-se que $50 \%$ dos idosos, com 80 anos ou mais, obtinham uma renda até meio salário mínimo, sendo que na faixa entre 60 a 69 anos este percentual foi de cerca de $35 \% 13$.

Quanto às condições de saúde a maioria dos idosos apresentou uma maior distribuição de enfermidades crônicas, sendo as mais citadas: hipertensão arterial e o diabetes mellitus. A presença de doenças crônicas simula um desafio à qualidade de vida dos idosos, por está diretamente ligada a incapacidade funcional, comprometendo sua independência e autonomia nas atividades diária ${ }^{14,15}$.

Observou-setambém do transcorrer doestudouma maior distribuição de idosos realizandotratamentomedicamentoso associado à fisioterapia, constatando-se que a reabilitação gerontológica vai auxiliar na preservação da função, adiamento da instalação de incapacidades, através de medidas preventivas, com o objetivo de reduzir o comprometimento imposto por incapacidades, promovendo um modo de vida mais saudável adaptando o indivíduo de maneira propícia ${ }^{15}$.

A maioria dos idosos avaliados neste estudo foram classificados como dependentes tanto nas ABVD, quanto nas AIVD. Acredita-se que este comprometimento possa estar relacionado à institucionalização, pois idosos comunitários conseguem realizar melhor suas atividades ${ }^{16}$. Por outro lado, os idosos que vivem em asilos, apresentam características importantes como aumento de sedentarismo, perda de autonomia, ausência de familiares, que entre outros, influência para 0 aumento das prevalências das morbidades e co-morbidades relacionadas à autonomia ${ }^{17}$. 
O processo de envelhecimento acarreta o declínio da aptidão física e da capacidade funcional, o que se agrava com o sedentarismo tornando- o cada vez mais dependente. Pesquisas mostram que há uma prevalência maior desta situação principalmente entre idosos institucionalizados, tornando esse declínio cada vez mais rápido e progressivo ${ }^{18}$.

O estilo de vida sedentário, combinado com o processo de envelhecimento, pode agravar a redução da força muscular inspiratória e expiratória, resultando na redução da pressão intratorácica e no fluxo expiratório durante a tosse. Constatou-se que os valores de PIMáx estão abaixo do limite normal e os valores de PEMáx estão dentro do limite previsto. Segundo estudos padrões, são considerados anormais e indicativos de alguma alteração da retidão da musculatura respiratória se encontrados na PIMáx e PEMáx valores abaixo de $60 \%$ do previsto ${ }^{17}$.

Foram analisados e conferidos: força muscular de idosos institucionalizados (PImáx $-57 \mathrm{cmH}_{2} \mathrm{O}$ e PEmáx de $70 \mathrm{cmH}_{2} \mathrm{O}$ ), com alunos (acima de 50 anos) da Universidade da Experiência (PImáx de $-72 \mathrm{cmH}_{2} \mathrm{O}$ e PEmáx de $97 \mathrm{cmH}_{2} \mathrm{O}$ ), e nesse artigo foi comprovado que as forças da musculatura respiratória eram menores nos idosos residentes em instituições de longa permanência ${ }^{16,17}$. Observou-se no presente estudo que os valores da PEmáx influenciam no comprometimento das atividades instrumentais de vida diária, demonstrando assim haver relação entre capacidade respiratória e funcionalidade em idosos residentes em instituição de longa permanência.

A redução da força dos músculos esqueléticos que ocorre com o avanço da idade, ocorre também nos músculos respiratórios podendo está relacionada com alterações de atividades e mudanças geométricas do tórax, diminuindo a massa muscular e o número de fibras, podendo comprometer também sua função ventilatória e não-ventilatória, estando relacionado com a tosse, fala e deglutição ${ }^{15,18}$.

O envelhecimento diminui a complacência da caixa torácica, reduzindo as forças de tração da parede torácica, contribuindo para a redução nas pressões inspiratórias e expiratórias, causando alterações da força muscular respiratória do idoso, sendo de grande importância para a diminuição da função pulmonar e são descritas como limitantes, comprometendo a sua capacidade funcional ${ }^{13,14}$.

A avaliação da relação da potência da musculatura respiratória com a capacidade funcional tem grande importância, podendo favorecer, introduzindo intervenções que irá proporcionar melhores condições aos idosos para as suas atividades de rotina, obtendo uma vida mais funcional ${ }^{18}$.

\section{CONSIDERAÇÕES FINAIS}

No presente estudo a análise mostrou uma correlação moderada, positiva e significativa entre o PEmáx e AIVD. Sendo verificado diferença estatística significativa entre os valores de PEmáx e o comprometimento das AIVD.

Estes dados sugerem que o comprometimento da musculatura expiratória em decorrência as AIVD e ao 
processo natural do envelhecimento, pode limitar a capacidade funcional em idosos residentes em Instituição de Longa Permanência. Dessa forma, faz-se necessário a inclusão do fortalecimento dessa musculatura. Os dados deste estudo indicam a necessidade de novas pesquisas.

\section{REFERÊNCIAS}

1. Gusmão MFS, Duarte SFP, Lago LS, Nascimento CP, Almeira RFF, Reis LA. Mensuração das pressões respiratórias máximas em idosos participantes de grupos de convivência. InterScientia. 2015;3(2):133-41.

2. Almeida RFF, Nascimento CP, Lago LS, Gusmão MFS, Duarte SFP, Reis LA. Relação entre força muscular respiratória e faixa etária em idosos participantes de grupos de convivência. Rev Enferm Contempor. 2015;4(1):33-8.

3. Farrareze M, Piccoli JCJ, Souza RM. Gordura corporal e função pulmonar: um estudo em idosas de Novo Hamburgo, RS, Brasil. Rev Univ Educ Fis Deporte. 2014; 7:69-75.

4. Reis LA, Moreira JSS, Duarte FP. Risk of falling, respiratory and functional capacity in elderly residents in institutions of long stay. Rev Neurocienc. 2015; 23(3):397-404.

5. Vasconcello JAC et al. Pressões respiratórias máximas e capacidade funcional em idosas assintomáticas. Fisioterapia em Movimento, 20(3), 2017.

6. Silva CM, Araujo A, Silva AL, Sousa V, Gomes Neto M, Saquetto M. Avaliação da força muscular respiratória e capacidade funcional em pacientes com fibrose cística. Acta Fisiátrica. 2016; 23(4): 186-190.

7. Bertolucci PHF et al. O Mini-Exame do Estado Mental em uma população geral: impacto da escolaridade. Arq. Neuropsiquiatr. 1994; 52 (1):1-7.

8. Black LF, Hyatt RE. Maximal respiratory pressures: normal values and relationship to age and sex. Am Rev Respir Dis. 1969;99(5):696-702.

9. Shah S, Vanclay F, Cooper B, Improving the sensitivity of the Barthel Index for stroke rehabilitation. J Clin Epidemiol. 1989; 42(8):703-9. 
10. Lawton MP, Brody EM. Assesment of older people: self-maintaining and instrumental activities of daily living. Gerontologist. 1969; (9):179-85

11. Almeida RFF, Nascimento CP, Lago LS, Gusmão MFS, Duarte SFP, Reis LA. Relação entre força muscular respiratória e faixa etária em idosos participantes de grupos de convivência. Revista Enfermagem Contemporânea. 2015;4(1):33-38.

12. Abrahin O, Rodrigues RP, Nascimento VC, Silva-Grigoletto MS, Sousa EC, Marçal AC. Single and multiple set resistance training improves skeletal and respiratory muscle strength in elderly women. Dovepress Journal. 2014;9:1775-1782.

13. Joshua AM, D'Souza V, Unnikrishnan B, Mithra P, Kamath A, Acharya V, et al. Effectiveness of Progressive Resistance Strength Training Versus Traditional Balance Exercise in Improving Balance Among the Elderly: a Randomised Controlled Trial. J ClinDiagn Res. 2014;8(3):98-102.

14. Monteiro DP, Brito R, Carvalho MLV, Montemezzo D, Parreira VF, Pereira DAG. Shuttle walking test as an instrument for assessment of functional capacity: a literature review. Ciência\&Saúde. 2014;7(2):92-97.

15. Ike D, Bueno CEC, CutlacNeto J, Pessoa-Santos BV, Jamami M. Analysis of agreement between peak expiratory flow meters and comparison of reference values. FisioterMov. 2017;30(3):509-517.

16. Baltieri L, Dos Santos LA, Furlan GN, Moreno MA. Respiratory muscle strength and thoracoabdominal mobility in sedentary elderly, adults and players of adapted volleyball: a pilot study. FisioterPesq 2014;21(4):314-319.

17. Nascimento VC, Teixeira RC, Sousa EC, Gomes ELFD, Costa D. Effect of resistance training on physical performance and respiratory functional capacity of elderly women. J. Phys. Educ. 2018; 29, e2943.

18. Borges et al. Envelhecimento e força muscular respiratória de idosos independentes residentes de uma instituição de longa permanência em regime aberto. JCBS. 2015; 1(2): 61-67. 